\title{
Comparison of surgical time and IOP spikes with two ophthalmic viscosurgical devices following Visian STAAR (ICL,V4c model) insertion in the immediate postoperative period
}

Clinical Ophthalmology

27 January 2016

Number of times this article has been viewed

\section{Sri Ganesh \\ Sheetal Brar \\ Department of Phaco and Refractive Surgeries, Nethradhama Superspeciality Eye Hospital, Bangalore, India}

Correspondence: Sheetal Brar Department of Phaco and Refractive Surgeries, Nethradhama Superspeciality Eye Hospital, 256/14, Kanakapura Main Road, 7th Block, Jayanagar, Bangalore 560082, India

Tel +91 9591002092

Email brar_sheetal@yahoo.co.in
Purpose: To compare the effect of two ocular viscosurgical devices (OVDs) on intraocular pressure (IOP) and surgical time in immediate postoperative period after bilateral implantable collamer lens (using the V4c model) implantation.

Methods: A total of 20 eligible patients were randomized to receive $2 \%$ hydroxypropylmethylcellulose (HPMC) in one eye and $1 \%$ hyaluronic acid in fellow eye. Time taken for complete removal of OVD and total surgical time were recorded. At the end of surgery, IOP was adjusted between 15 and $20 \mathrm{mmHg}$ in both the eyes.

Results: Mean time for complete OVD evacuation and total surgical time were significantly higher in the HPMC group $(P=0.00)$. Four eyes in the HPMC group had IOP spike, requiring treatment. IOP values with noncontact tonometry at 1, 2, 4, 24, and 48 hours were not statistically significant $(P>0.05)$ for both the groups.

Conclusion: The study concluded that $1 \%$ hyaluronic acid significantly reduces total surgical time, and incidence of acute spikes may be lower compared to $2 \%$ HPMC when used for implantable collamer lens (V4c model).

Keywords: OVD, hyaluronic acid, ICL, V4c, IOP spikes

\section{Introduction}

Various ocular viscosurgical devices (OVDs) have been investigated and compared for their utility as space maintainers, ease of aspiration, endothelial protection, and postoperative intraocular pressure (IOP) changes after cataract surgery. ${ }^{1,2}$ However, no comparison study between two OVDs in Visian STAAR Implantable Collamer Lens (ICL) establishing advantages of one OVD over the other has been reported till date. The present study hypothesized that intraoperative use of high-viscosity, cohesive hyaluronic acid (HA)-based OVD in the new Visian V4c ICL with CentraFLOW technology may facilitate its quick, easy, and complete removal from anterior chamber, minimizing IOP spikes in the immediate postoperative period when compared to $2 \%$ hydroxypropylmethylcellulose (HPMC), a low-viscosity dispersive OVD.

\section{Materials and methods}

The institutional ethics committee of Nethradhama Superspeciality Eye Hospital approved the study and the study adhered to the tenets of the Declaration of Helsinki. Written informed consent was obtained from all the patients participating in the trial. Preoperative examination included assessment of uncorrected and corrected distance visual acuity, subjective and cycloplegic refraction, anterior segment examination 
with slit lamp biomicroscopy, noncontact tonometry (NCT) (Nidek NT-510; Gamagori, Japan), indirect ophthalmoscopy, topography (Orbscan IIz, Bausch \& Lomb Incorporated, Bridgewater, NJ, USA), dry eye assessment (Schirmer I test and tear film breakup time), and specular microscopy (Tomey, Nagoya, Japan) for endothelial cell density (ECD).

This trial was registered with the Clinical Trial Registry of India, CTRI/2014/05/0004594.

\section{Inclusion criteria}

The study included individuals aged 21 years and above with stable refraction over past 12 months, myopia or myopic astigmatism amenable to correction with V4c Visian ICL or V4c Toric ICL anterior chamber depth $>2.8 \mathrm{~mm}$ with Orbscan and minimum ECD of 1,500 cells $/ \mathrm{mm}^{2}$, willingness to sign consent form, and ensured follow-up.

\section{Exclusion criteria}

Exclusion criteria included age $<21$ years, progressive myopia, unstable refraction, ectatic corneal disorders such as keratoconus or pellucid marginal degeneration, chronic inflammatory anterior or posterior segment pathology, known cases of glaucoma, severe ocular surface pathology, inadequate anterior chamber depth $<2.8 \mathrm{~mm}$, and low endothelial cell counts $\left(<1,500\right.$ cells $\left./ \mathrm{mm}^{2}\right)$.

Both the eyes of patients undergoing bilateral surgery with Visian STAAR ICL or V4c Toric ICL model for correction of myopia and myopic astigmatism were randomized to receive 2\% HPMC (Viscomet PF; Sun Pharmaceuticals, Bangalore, India) in one eye (Group 1) and 1\% HA (Hyal 2000'TM; LG Life Sciences, Seoul, Korea) in the fellow eye (Group 2). Randomization of eyes was done using computergenerated random numbers. No attempt was made to mask the randomization of OVD during the operation due to different handling characteristics of the two agents.

\section{Surgical techniques}

All surgeries were performed by a single experienced surgeon (SG) under topical anesthesia. The surgical steps from temporal $2.8 \mathrm{~mm}$ clear corneal wound creation to insertion of ICL were similar in both the eyes. Total surgical time from the creation of the corneal wounds to hydration of the same was recorded in minutes. After the insertion of footplates of ICL into the ciliary sulcus, time taken for complete removal of OVD from the anterior chamber was noted. Aspiration was performed with automated I/A coaxial probe with a flow rate of $60 \mathrm{~cm}^{3} / \mathrm{min}$ and vacuum of $600 \mathrm{mmHg}$ in both the groups. The aspiration port was directed toward the central hole (Figure 1). In the HPMC

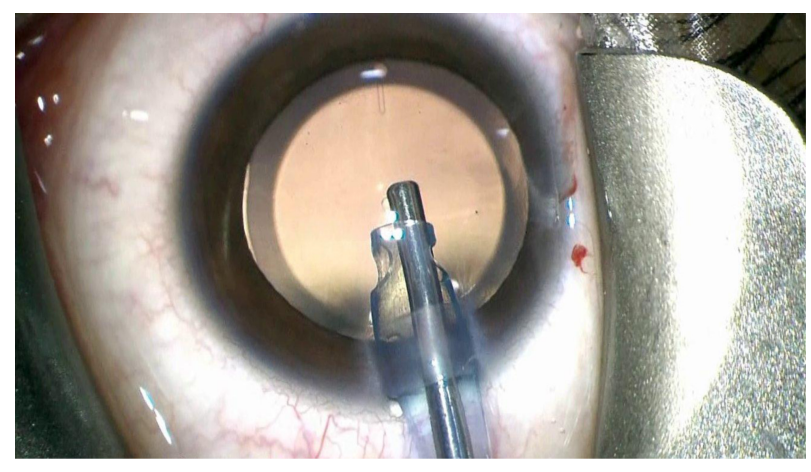

Figure I Figure showing aspiration of OVD using a coaxial irrigation aspiration probe directed toward the central hole of the Visian STAAR ICL.

Abbreviations: ICL, implantable collamer lens; OVD, ocular viscosurgical device.

group, aspiration was continued until a flower petal pattern was visible underneath the ICL, indicating that most of the OVD was aspirated. In the HA group, surgeon visualization of evacuation of HA as bolus was taken as the end point.

At the end of surgery, IOP was adjusted at $15 \mathrm{mmHg}$ for both the eyes with Schiotz tonometer; Reichert Technologies, New York, NY, USA. Miotics were not used for pupillary constriction in either group at the end of surgery.

Patients were monitored for symptoms and IOP with NCT at 1, 2, 4, 24, and 48 hours after surgery, the examiner being masked with respect to the groups. Applanation tonometry was not used in the preoperative and immediate postoperative periods to avoid instillation of topical anesthetic drops and fluorescence dye, which may cause epithelial edema and trauma and thus increase patient discomfort. Postoperatively, antibiotic steroid combination eye drops (Oflacin-DX; Micro Labs, Bangalore, India) were prescribed four times for 2 weeks. No antiglaucoma medication (oral/topical) was prescribed unless indicated.

Statistical analysis was performed using the SPSS software for Windows (version 20.0.0, IBM Corporation, Armonk, NY, USA). To compare the data between the two groups, paired $t$-tests were used. $P$-value $<0.05$ was considered significant.

\section{Results}

A total of 20 patients, ten males and ten females with a mean age $28 \pm 7.1$ years (range: $18-45$ years), were enrolled in the study. Preoperative, intraoperative, and postoperative data of the two groups are summarized in Table 1. Preoperatively, the mean spherical equivalents in Group 1 and Group 2 were $-10.9 \pm 4.52$ and $-9.5 \pm 3.61 \mathrm{D}$, respectively, which were statistically significant. In terms of corrected distant visual acuity, ECD and mean IOP, both the groups showed similar results $(P>0.05)$. 
Table I Preoperative, intraoperative, and postoperative parameters of Group I ( $\mathrm{n}=20$ eyes) and Group 2 ( $\mathrm{n}=20$ eyes)

\begin{tabular}{llll}
\hline Parameters & Group I (2\% HPMC) & Group 2 (I\% HA) & P-value \\
\hline Preoperative parameters & & & \\
SE & $-10.9 \pm 4.52$ & $-9.5 \pm 3.61$ & 0.04 \\
CDVA (LogMAR) & $0.10 \pm 0.130$ & $0.10 \pm 0.162$ & 1.00 \\
ECD (cells $\left./ \mathrm{mm}^{2}\right)$ & $3,101.9 \pm 308.34$ & $3,129.0 \pm 280.77$ & 0.30 \\
IOP NCT (mmHg) & $14.7 \pm 3.1$ & $15.0 \pm 2.9$ & 0.42 \\
Intra- and immediate postoperative parameters & $17.3 \pm 0.3$ & $17.3 \pm 0.2$ & 0.57 \\
IOP at the end of surgery $(\mathrm{mmHg})$ & $23.2 \pm 7.9$ & $21.2 \pm 5.0$ & 0.14 \\
IOP at I hour $(\mathrm{mmHg})(\mathrm{NCT})$ & $23.3 \pm 9.3$ & $19.2 \pm 5.3$ & 0.06 \\
IOP at 2 hours $(\mathrm{mmHg})(\mathrm{NCT})$ & $15.8 \pm 3.9$ & $15.0 \pm 3.3$ & 0.36 \\
IOP at 4 hours $(\mathrm{mmHg})(\mathrm{NCT})$ & $14.7 \pm 4.1$ & $14.8 \pm 4.3$ & 0.46 \\
IOP at 24 hours $(\mathrm{mmHg})(\mathrm{NCT})$ & & & \\
\hline
\end{tabular}

Note: Data presented as mean \pm SD (with the exception of $P$-values).

Abbreviations: CDVA, corrected distance visual acuity; HA, hyaluronic acid; HPMC, hydroxypropylmethylcellulose; ECD, endothelial cell density; IOP, intraocular pressure; LogMAR, log of the minimum angle of resolution; NCT, noncontact tonometry; SD, standard deviation; SE, spherical equivalent.

Intraoperatively, the mean time taken for complete removal of OVD from the anterior chamber was 2.4 minutes in Group 1 and 0.3 minutes in Group 2, which was also statistically significant $(P<0.01)$ (Figure 2$)$.

In all, $100 \%$ (20) eyes in Group 1 and $60 \%$ (12) eyes in Group 2 had transient increase in IOP $(>22 \mathrm{mmHg})$ within the first 24 hours, which settled by 48 hours. However, mean IOP values between the two groups at 1,2,4,24, and 48 hours were not statistically significant ( $P$-values: $0.14,0.06,0.36$, 0.46 , and 0.88 , respectively) (Table 1$)$.

The results were clinically significant at the 2 nd hour. At the 2nd hour, four eyes in Group 1 had IOP spike, ie, IOP $>30 \mathrm{mmHg}$ (range: $33-45 \mathrm{mmHg}$ ) (Figure 2). These patients did not complain of systemic symptoms such as headache, nausea, or vomiting. Slit lamp examination at the time of acute spike did not reveal any structural abnormality other than diffuse epithelial edema in the one eye with an IOP of $45 \mathrm{mmHg}$. All patients were prescribed topical $2 \%$ dorzolamide (Dorzox; Cipla, Mumbai, India) three times per day. One eye with an IOP of $45 \mathrm{mmHg}$ was additionally subjected to burping of wound with a 26-gauge needle to express some fluid from the anterior chamber to bring down the IOP. IOP normalized with treatment within 24 hours in all the four eyes. None of the eyes in Group 2 showed any such adverse effects.
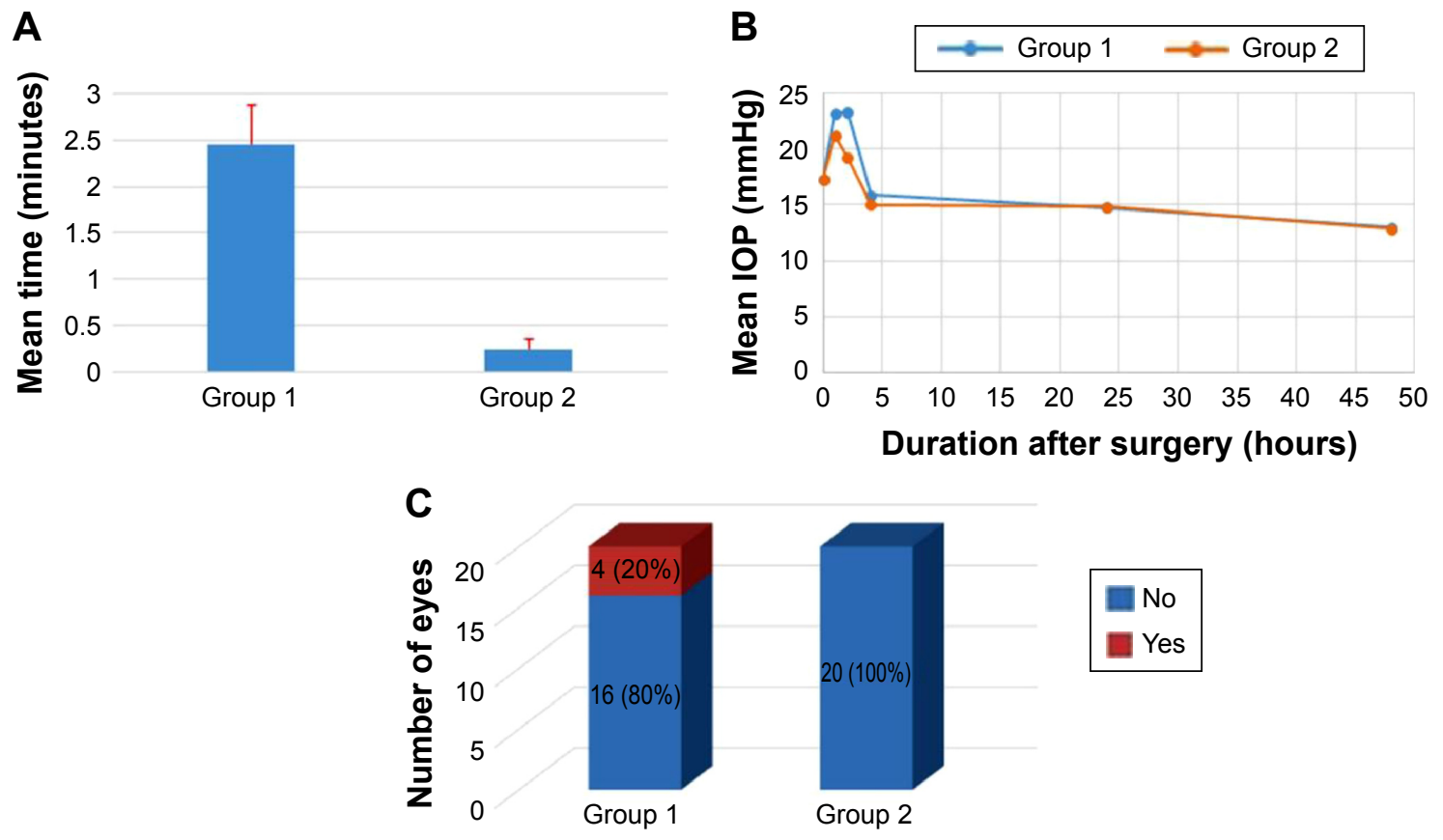

Figure 2 Comparison between Group I and Group 2.

Notes: Comparison in terms of (A) total surgical time, (B) mean IOP at I, 2, 4, 24, and 48 hours, and (C) number of eyes with IOP $>30-45$ mmHg within 24 hours. Group I (2\% HPMC); Group 2 (I\% HA).

Abbreviation: IOP, intraocular pressure. 
At 15 days postoperatively, IOP (NCT), uncorrected visual acuity, postoperative vault on AS-OCT (Optovue, Fremont, CA, USA), and ECD were comparable between both the groups $(P>0.05)$ (Table 1$)$. None of the eyes developed steroid-induced response requiring long-term antiglaucoma medication.

\section{Discussion}

The Visian ICL posterior chamber phakic intraocular lens is shown to be a valuable surgical option to treat ametropia of various refractive ranges. ${ }^{3}$ Many studies have reported increased IOP levels after ICL implantation. Incomplete removal of viscoelastic material is one of the causes of immediate IOP rise after ICL surgery apart from steroid use, pigment dispersion, and pupillary block. ${ }^{4}$

In the Visian ICL V4b model, peripheral iridotomies, either preoperatively or at the time of surgery, are required to prevent pupillary block and sudden IOP rise in the immediate postoperative period. The recently introduced Visian ICL V4c with CentraFLOW technology has a hole in the center of ICL optic, called as the KS-AquaPORT, which is meant to improve aqueous humor circulation in the eye. This new development has eliminated the need for peripheral iridectomy, thus simplifying the surgical procedure and significantly reducing the complications associated with iridotomy. ${ }^{5}$

The manufacturers of Visian STAAR ICL recommend the use of biologically inactive methylcellulose instead of active HA-based OVD with ICL for loading and intraoperative use. This may be due to the fact that HA has been shown to influence hydration and physical properties of tissues and is known to interact with other extracellular matrix macromolecules that are essential to the structure of several tissues. ${ }^{6}$ Hence, it is theorized that the remaining HA between ICL and crystalline lens may form a viscoelastic putty clot over time and compromise intraocular structures in several ways. However, there are no data in literature to support this theory due to the lack of comparative studies between the two OVDs in ICL. Also, this may be relevant to the earlier available V4b model, which is devoid of a central hole.

Sodium hyaluronate and its new-generation compounds have become indispensible tools for intraocular surgeries due to favorable rheological properties such as cohesiveness, elasticity, high-viscosity, and high pseudoplasticity. ${ }^{7,8}$ There are numerous comparative studies in cataract surgery suggesting that HA-based OVDs are better than dispersive OVDs due to excellent space maintenance and ease in injection and removal from the eye. They are also being preferably used in keratoplasty, glaucoma, and retinal surgeries due to various advantages. ${ }^{9,10}$

However, no such comparison studies have been published for ICL since the intraoperative use of HA has not been encouraged due to fear of potential tissue interactions, toxicity, and acute glaucoma when retained in the eye..$^{6,11}$

In this study, the rheological properties of $1 \%$ HA were exploited well in the V4c model with KS-AquaPORT. Significant reduction in aspiration time leading to reduction in overall surgical time was noticed, as seen in similar studies with cataract. ${ }^{9}$ Although not statistically significant, four eyes in the HPMC group had acute IOP spike requiring treatment. Steroid response in these eyes was ruled out, as the contralateral eye ( $1 \% \mathrm{HA})$ did not show corresponding increase in IOP. There were no significant differences in clinical outcomes and ECD when compared to HPMC. However, comparisons with dilutions of HA other than $1 \%$ have not been done.

Results of this trial suggest that it is feasible to use 1\% HA intraoperatively in routine clinical practice for the Visian STAAR V4c model due to favorable clinical outcomes in terms of surgical time and incidence of postoperative rise in IOP when compared to $2 \%$ HPMC. These findings may be further verified by studies with larger sample size and longer follow-up duration.

\section{Acknowledgment}

This study did not receive funding from any source.

\section{Disclosure}

The authors report no conflicts of interest in this work.

\section{References}

1. Assia EI, Apple DJ, Lim ES, Morgan RC, Tsai JC. Removal of viscoelastic materials after experimental cataract surgery in vitro. J Cataract Refract Surg. 1992;18(1):3-6.

2. Koch DD, Liu JF, Glasser DB, Merin LM, Haft E. A comparison of corneal endothelial changes after use of Healon or Viscoat during phacoemulsification. Am J Ophthalmol. 1993;115(2):188-201.

3. Sanders DR, Doney K, Poco M; ICL in Treatment of Myopia Study Group. United States Food and Drug Administration clinical trial of the implantable collamer lens (ICL) for moderate to high myopia: three-year follow-up. Ophthalmology. 2004;111(9):1683-1692.

4. Fernandes P, González-Méijome JM, Madrid-Costa D, Ferrer-Blasco T, Jorge J, Montés-Micó R. Implantable collamer posterior chamber intraocular lenses: a review of potential complications. J Refract Surg. 2011;27(10):765-776.

5. Higueras-Esteban A, Ortiz-Gomariz A, Gutiérrez-Ortega R, et al. Intraocular pressure after implantation of the Visian Implantable Collamer Lens With CentraFLOW without iridotomy. Am J Ophthalmol. 2013;156:800-805.

6. Necas J, Bartosikova L, Brauner P, Kolar J. Hyaluronic acid (hyaluronan): a review. Veterinarni Medicina. 2008;53(8):397-411. 
7. Bothner H, Wik O. Rheology of hyaluronate. Acta Otolaryngol. 1987; 442(suppl):25-30.

8. Higashide T, Sugiyama K. Use of viscoelastic substance in ophthalmic surgery-focus on sodium hyaluronate. Clin Ophthalmol. 2008;2(1): 21-30.

9. Bissen-Miyajima H. In vitro behavior of ophthalmic viscosurgical devices during phacoemulsification. J Cataract Refract Surg. 2006; 32(6):1026-1031.
10. Hutz WW, Eckhardt HB, Kohnen T. Comparison of viscoelastic substances in phacoemulsification. J Cataract Refract Surg. 1996;22(7): 955-959.

11. Lindquist TD, Edenfield MC. Cytotoxicity of viscoelastics on cultured corneal epithelial cells measured by plasminogen activator release. J Refract Corneal Surg. 1994;10(2):95-102.

\section{Publish your work in this journal}

Clinical Ophthalmology is an international, peer-reviewed journal covering all subspecialties within ophthalmology. Key topics include: Optometry; Visual science; Pharmacology and drug therapy in eye diseases; Basic Sciences; Primary and Secondary eye care; Patient Safety and Quality of Care Improvements. This journal is indexed on

Submit your manuscript here: http://www.dovepress.com/clinical-ophthalmology-journal

\section{Dovepress}

PubMed Central and CAS, and is the official journal of The Society of Clinical Ophthalmology (SCO). The manuscript management system is completely online and includes a very quick and fair peer-review system, which is all easy to use. Visit http://www.dovepress.com/ testimonials.php to read real quotes from published authors. 